\title{
Review
}

\section{A Novel Hospital-Based Mass Casualty Decontamination Facility for Hazardous Material Disasters}

\author{
Ponampalam R, MBBS, FRCS, FAMS, GDOM* \\ Department of Emergency Medicine, Singapore General Hospital, Outram Road 169608, Singapore \\ "Corresponding author \\ Ponampalam R, MBBS, FRCS, FAMS, GDOM \\ Senior Consultant, Department of Emergency Medicine, Singapore General Hospital, Outram Road I69608, Singapore; E-mail: ponampalam@singhealth.com.sg
}

\section{Article information}

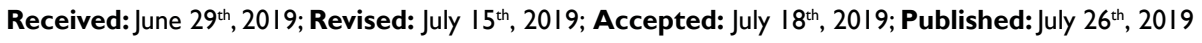

\section{Cite this article}

Ponampalam R.A novel hospital based mass casualty decontamination facility for hazardous material disasters. Toxicol Forensic Med Open J. 2019;4(I): 18-23. doi: $10.17140 /$ TFMOJ-4-129

\begin{abstract}
|
Since the Sarin incident in the subways of Tokyo in 1995, there has been an unprecedented increase in the use of chemical agents on civilian populations internationally. This scourge of chemical terrorism has been relentless worldwide and is likely to continue to be a public health issue that needs to be addressed by the relevant authorities as part of national disaster preparedness and response. One aspect of chemical disasters involves the need for mass decontamination of chemically-contaminated casualties from the scene. The traditional role of hazardous materials civil defence experts in providing such decontamination of victims in the pre-hospital setting is limited by many factors. The presence of congestion in densely populated areas in a highly built up environment of modern-day cities, compounds the timeliness of putting up cordons and crowd control and hence delays the prompt set up of such mobile decontamination facilities close to the incident site. The expected side effect is an almost instantaneous influx of contaminated casualties to the nearest hospital in such situations, which drives the need for public hospitals to be ultimately capable of performing mass casualty decontamination as part of hazardous materials disaster preparedness. This review presents an innovatively designed rapidly deployable hospital-based decontamination facility that has served a tertiary care hospital in Singapore for the last 2 decades in being prepared for managing mass casualties arriving from a chemical disaster in a timely manner.
\end{abstract}

\section{Keywords}

Decontamination; Chemical incident; Industrial disasters; Toxic industrial chemicals; Hazardous materials preparedness; Disaster contingency plans; Emergency preparedness.

\section{INTRODUCTION}

$\mathrm{D}$ isasters involving chemical release are of concern in our modern industrialized world with highly urbanized cities because of the propensity of such incidents for causing injury and death in large numbers such as the Bhopal disaster ${ }^{1}$ in India in 1984. In our present times of prevailing low intensity conflicts and terrorism-linked events, there is an increasing necessity for medical preparedness in dealing with these situations. The Sarin Incident in Tokyo and Matsumoto, ${ }^{2-4}$ demonstrated the impact of mass casualty incidents from chemical terrorism using chemical warfare agents released by simple improvised chemical dispersion methods on an unsuspecting civilian population. Most patients from this incident presented promptly to the nearest emergency departments (ED) with no pre-hospital or hospital decontamination resulting in significant secondary contamination of emergency rescue and hospital personnel. Our local experience ${ }^{5-7}$ in chemical disasters demonstrated the impact on hospital personnel when over a third of healthcare providers on duty including all responding trauma team members developed secondary symptoms from managing tear gas contaminated casualties with no prior decontamination. Bearing this in mind, all public hospitals in Singapore were tasked by the health authorities to prepare for hazardous materials disasters and had to be equipped to deal with managing contaminated casualties and the decontamination of these victims to reduce toxic exposure to the victims and address the potential spread of contamination to limit damage from secondary exposure of healthcare providers and secondary contamination of healthcare facilities. Most healthcare systems depend on the fire services which predominantly uses the traditional ladder pipe system set up for decontamination of mass casualties. ${ }^{8}$ There have been innovative approaches to dealing with this situation including a recent study 
Imamedjian et $\mathrm{al}^{9}$ trialling the use of public buses and tents as holding areas and decontamination facility. The stark challenges of acute care hospitals in the United Kingdom ${ }^{10}$ to be prepared to perform mass decontamination are well-documented from lack of critical infrastructure, logistics, manpower and consistent regular training to maintain competency amongst healthcare providers. In the Singapore context, we have been using our tried and tested rapid deployment hospital decontamination station (HDS) located near to the ED for the last two decades. This article reviews the unique features of this entity.

\section{Unique Features of the Hospital Decontamination Station (HDS)}

Singapore General Hospital (SGH), was the first institution in Singapore to take on the challenge to innovate and build the HDS which set the precedent for all other local institutions. The Department of Emergency Medicine (DEM) celebrated its $70^{\text {th }}$ anniversary in 2018. It is an opportune moment to acknowledge one of its pioneer achievement's; the hospital decontamination station (HDS), an important innovative facility the first of its kind in Singapore. The HDS was built in the early part of this millennium and was intended as a medical countermeasure to the rising threat of chemical terrorism and industrial chemical accidents to decontaminate chemically contaminated casualties arriving at the hospital from the disaster scene at short notice. We describe the special considerations that went into constructing the HDS that makes it stand out as a significant countermeasure addressing the unique challenges of dealing with hazardous materials disasters with contaminated casualties by its timely deployment.

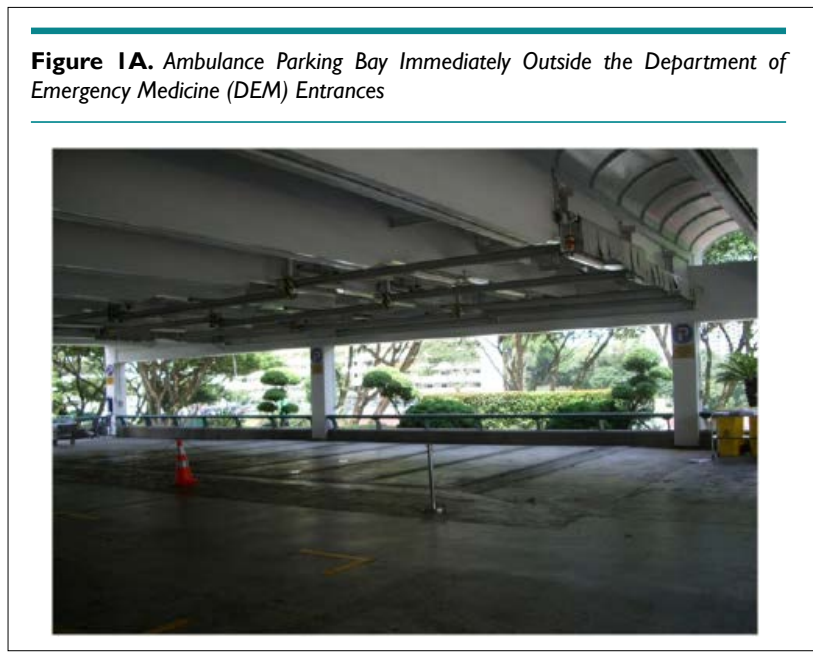

Lessons from the past, both local and overseas, have shown the rapid escalation of chemical disasters with mass casualties. The likelihood of setting up pre-hospital decontamination during disasters by rescue services in most modern-day built up cities which are gridlocked with traffic congestion and high population density is low. It is appreciated that prompt deployment of such decontamination facilities to deal with contaminated casualties who are likely to arrive in large numbers at short notice with no prior decontamination at scene is paramount to successful management of such scenarios. In this regard,the HDS (Figures 1A, 1B and 1C), a semi-automatic decontamination facility located in proximity just outside the entrance of the Emergency Department (ED) that could be operational within 5-minutes of activation by a single staff member is purpose fit. The alternative approach in the past using mobile commercial decontamination systems (Figure 2) took 45-minutes to deploy with specially trained manpower. The space constraints in storing these commercial decontamination sets, retrieving it when required for deployment and short shelf life are additional limitations.
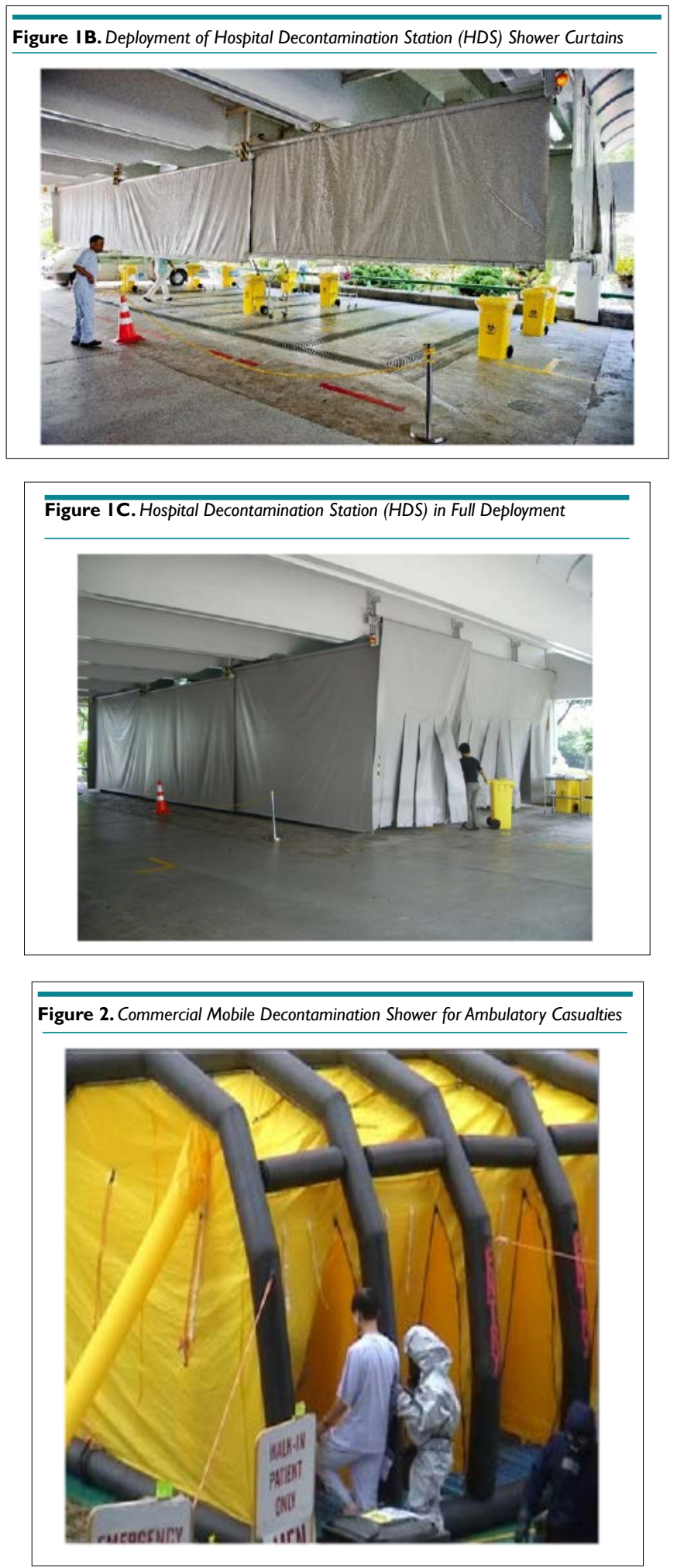
provides initial manpower to operate the system.

3. Rapid deployment (within 5-minutes of activation) by one staff member operating a control panel with clearly displayed instructions that are easy to follow (Figure 4).

4. Scalable deployment (partial or full) according to caseload depending on small or large scale incident (i.e. the number of lanes deployed can be selected) giving better control over limited space.

5. The enclosed part of the HDS is separated into 3 sections, disrobing area for removal of contaminated clothing, shower section and drying and re-clothing section. This allows for privacy while casualties are undergoing decontamination.

6. Separate sections for ambulatory and non-ambulatory casualties with clear markings on the floor to indicate direction of casualty flow to facilitate work processes.

7. Self-contained disrobing, shower and re-clothing compartment formed by flexible longitudinal screens (premature ventricular contractions (PVC) coated polyester yarn with counterweights and anchors at base to prevent movement) and specially designed lateral cut up staggered screens ensuring ease of movement of casualties and staff working in the HDS.

8. Dedicated, self-contained ventilation system (exhaust fans) which suctions air from within the decontamination station, passing through filters before blowing out to the atmosphere facilitating cooling for staff working with protective suits and reducing contamination within the confined space of the HDS in an environmentally friendly manner.

9. In-built, showers spray heads and soap dispensers deployed from the ceiling to facilitate decontamination, maximize space utilization and ensure decontamination crew safety (Figure 5) by reducing clutter and obstacles which predisposes to fall hazards.

2. Close proximity to ED, which operates round the clock and
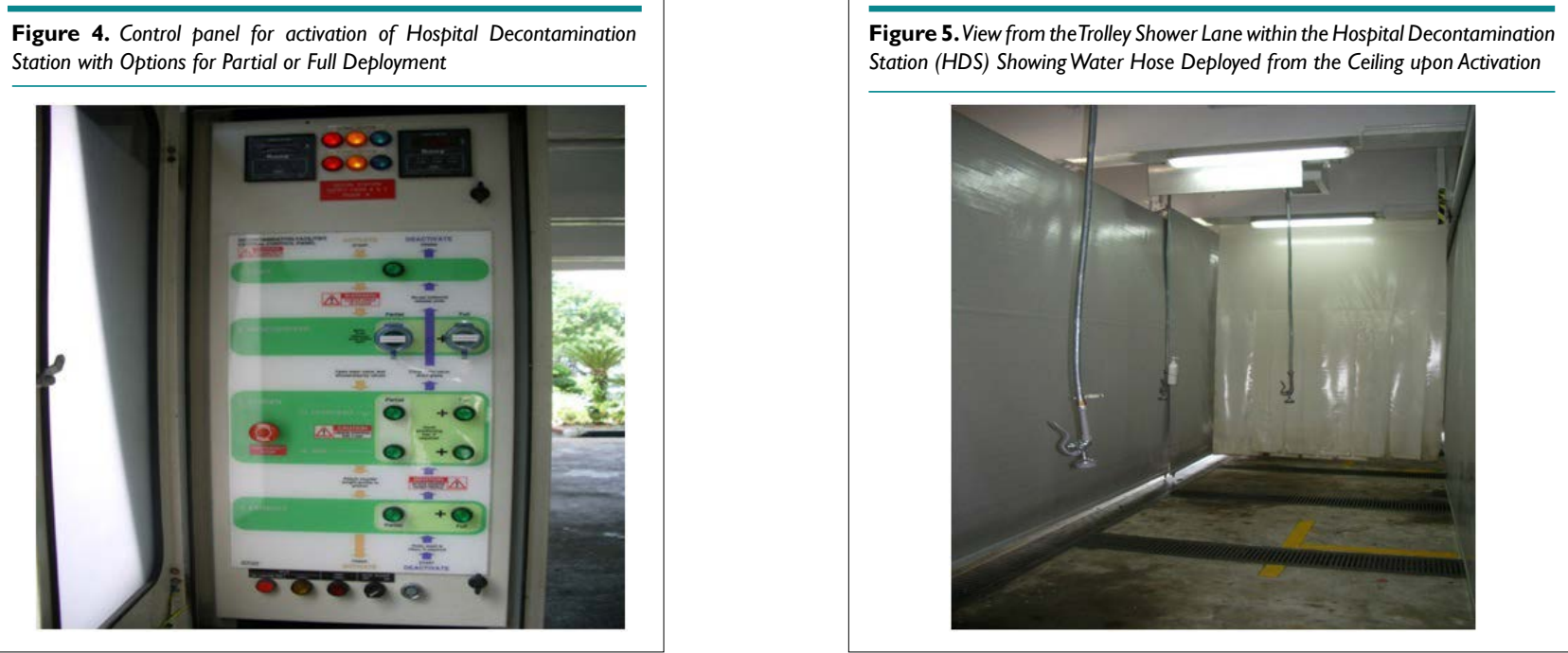
10. A large holding tank located beneath the HDS to contain the decontamination effluents for subsequent analysis and testing to determine safety for release into the sewerage system ensures water pollution control. This serves to conserve the environment.

\section{Utility of the Hospital Decontamination Station (HDS)}

Since its construction, the HDS has been used for several chemical incidents involving industrial chemical contaminated casualties and accidental toxic release situations. ${ }^{5-7}$ The rapid deployment capability allowed the incidents to be promptly managed preventing escalation of the incident with good outcomes.

The HDS has also been deployed successfully many times a year for both routine training as well as hospital and national disaster preparedness exercises (Table 1).

\begin{tabular}{|c|c|c|}
\hline Year & HDS Training Courses & HDS Chemical Disaster Exercises \\
\hline 2012 & 8 & I \\
\hline 2013 & 6 & I \\
\hline 2014 & 10 & 1 \\
\hline 2015 & 11 & - \\
\hline 2016 & 13 & 2 \\
\hline 2017 & 18 & 2 \\
\hline 2018 & 23 & 2 \\
\hline
\end{tabular}

\section{DISCUSSION}

A significant difference between hazardous materials incidents (chemical disasters) and conventional disasters involve the presence of chemical contamination in the environment as well as on casualties arriving from the incident site. This predisposes to ongoing chemical toxicity to the casualty as well as poses added risk to the rescuers and healthcare personnel dealing with such contaminated casualties.

In the Sarin incident in Tokyo in 1995, it was noted that approximately $9.9 \%$ of rescue workers ${ }^{2}$ (emergency medical technicians (EMT')) and $23 \%$ of hospital staff ${ }^{3}$ dealing with these chemical contaminated casualties suffered from symptoms due to secondary exposure from chemicals off gassing from clothing's of the casualties. Similar problems with secondary exposures amongst rescue personnel and hospital staff was noted in a similar attack ${ }^{4}$ involving Sarin in Matsumoto, Japan in 1994. It is noteworthy that no field or hospital decontamination of casualties was done in both these incidents.

Our own local experience, ${ }^{5-7}$ from treating casualties arriving from accidental toxic exposure has reinforced the potential risk of secondary exposure of healthcare workers from hazardous materials and has demonstrated the benefits of timely decontamination.
Hence, in chemical disasters it is of paramount importance to decontaminate all casualties so as to reduce the toxic effects on the casualty as well as to decrease the risk of secondary transmission. The latter is important to reduce the escalation of the event by spread of contamination and to reduce the loss of limited rescue and healthcare resources at a time of increased demand in a disaster situations. ${ }^{11}$ The basis for this has been recognized and there is guidance from occupational safety and health administration (OSHA) ${ }^{12}$ on best practices to follow in such situations. SGH has adapted and evolved its hazardous materials response contingency plans over time incorporating specific decontamination protocols into their disaster response plans ${ }^{13}$ (Figure 6).

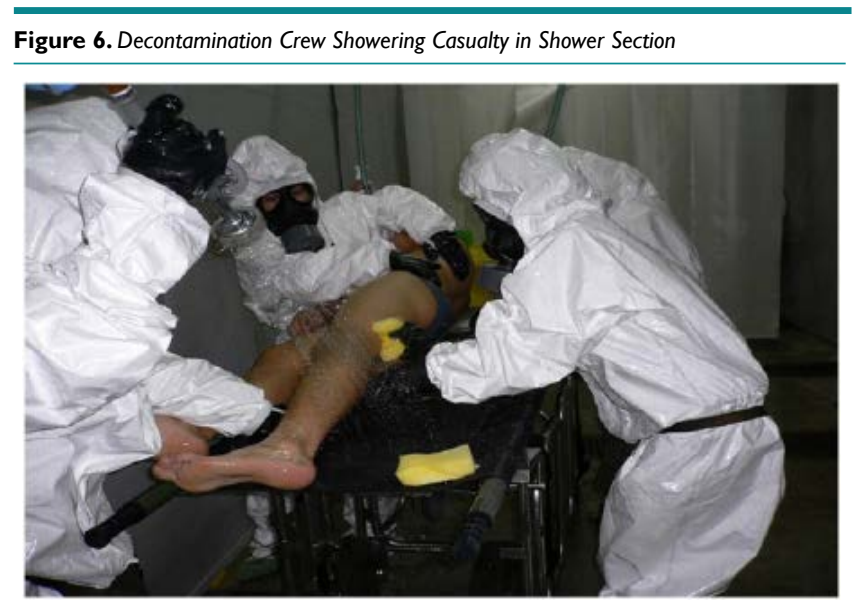

There are many recommendations for hospital-based decontamination. ${ }^{14-20}$ However, there is limited information on the specific infrastructure required and the necessary manpower and logistical support to execute a successful hospital-based decontamination system. There are many areas that need to be addressed for hospitals to be prepared to deal with hazardous materials mass casualty incidents ${ }^{21-24}$ and it is hoped that by sharing our experience on the specifics of one of our crucial assets, others would be able to gain some insights into what it entails and what needs to be done to be ready when the need arises.

\section{CONCLUSION}

The HDS marks an important milestone in the hospital's preparedness in dealing with hazardous materials disaster management. It is expected to be the primary decontamination facility for contaminated casualties from the incident site who make their way promptly to the hospitals ED bypassing onsite decontamination. With ED and hospital staff trained in deployment and operations of the HDS it will no doubt be an important countermeasure in the armamentarium for managing contaminated casualties and in the hospital's hazardous materials incident contingency response plans for many more years to come.

\section{ACKNOWLEDGEMENTS}

Figures presented in this article belong to the author. 


\section{REFERENCES}

1. Varma R, Varma DR. The Bhopal Disaster of 1984. Bulletin of Science, Technology \& Society. 2005; 25(1): 37-45. doi: $10.1177 / 0270467604273822$

2. Okumura T, Suzuki K, Fukuda A, et al. The Tokyo subway sarin attack: Disaster management, Part 1: Community emergency response. Acad Emerg Med. 1998; 5(6): 613-617. doi: 10.1111/j.15532712.1998.tb02470.x

3. Okumura T, Suzuki K, Fukuda A, et al. The Tokyo subway sarin attack: Disaster management, Part 2: Community emergency response. Acad Emerg Med. 1998; 5(6): 618-624.

4. Okudera H, Morita H, Iwashita T, et al. Unexpected nerve gas exposure in the city of matsumoto: Report of rescue activity in the first sarin gas terrorism. Am J Em Med. 1997; 15(5): 527-528. doi: 10.1016/S0735-6757(97)90201-1

5. Ponampalam R, Leong KF, Lim SH. Are we prepared to deal with hazardous material incidents? - An SGH perspective. SGH Proceedings. 2002; 11(4): 238-241.

6. Ngo SYA, Ponampalam R, Leong KF, Lim SH. Chlorine and its impact on an emergency department. Prehosp Disaster Med. 2007; 22(2): 136-139. doi: 10.1017/S1049023X00004519

7. Oh JJ, Yong R, Ponampalam R, Anantharaman V, Lim SH. Mass casualty incident involving pepper spray exposure: Impact on the Emergency Department and management of casualties. Hong Kong J Emerg Med. 2010; 17(4): 352-359. doi: 10.1177/102490791001700407

8. Power S, Symons C, Carter H, et al. Mass casualty decontamination in the United States: An online survey of current practice. Health Secur. 2016; 14 (4): 226-236. doi: 10.1089/hs.2016.0047

9. Imamedjian I, Maghraby NHM, Homier V. A hospital mass casualty exercise using city buses and a tent as a hybrid system for patient decontamination. Am J Disaster Med. 2017; 12(3): 189-196. doi: $10.5055 /$ ajdm.2017.0273

10. Clarke SF, Chilcott RP, Wilson JC, Kamanyire R, Baker DJ, Hallett A. Decontamination of multiple casualties who are chemically contaminated: A challenge for acute hospitals. Prehosp Disaster Med. 2008; 23(2): 175-181. doi: 10.1017/S1049023X00005811

11. Koenig KL, Boatright CJ, Hancock JA, et al. Health care facility-based decontamination of victims exposed to chemical, biological, and radiological materials. Am Journal of Emerg Med. 2008; 26(1): 71-80. doi: 10.1016/j.ajem.2007.07.004

12. Occupational Safety and Health Administration. OSHA best practices for hospital- based first receivers of victims from mass casualty incidents involving the release of hazardous substances. Web site. http://www.osha.gov/dts/osta/bestpractices/html/ hospital_firstreceivers.html. 2005. Accessed June 19, 2019.

13. Ponampalam R. Decontamination ó rationale and step-by-step procedure. SGH Proceedings. 2003, 12(4): 213-221.

14. Patient decontamination in a mass chemical exposure incident: National planning guidance for communities. US department of health and human services, office of the assistant secretary for preparedness and response. Website. http://www.phe.gov/Preparedness/responders/Documents/patient-decon-natl-plng-guide.pdf. Accessed June 19, 2019.

15. Primary response incident scene management (PRISM) guidance. US department of health and human services, office of the assistant secretary for preparedness and response. Website. https://www.medicalcountermeasures.gov/barda/cbrn/prism/. Accessed June 19, 2019.

16. 2012 Public Health Emergency Medical Countermeasures Enterprise (PHEMCE) Strategy. US Department of Health and Human Services, Office of the Assistant Secretary for Preparedness and Response website. http://www.phe.gov/Preparedness/mcm/ phemce/Documents/2012-PHEMCE-Strategy.pdf. Accessed June 19, 2019.

17. Lake WA, Divarco S, Schultz P, Gougelet R. Edgewood chemical biological center, and chemical, biological, radiological and nuclear school. Guidelines for mass casualty decontamination during a HAZMAT/weapon of mass destruction incident: Volumes 1 and 2. Web site. https://www.nfpa.org/ /media/files/news-andresearch/resources/external-links/first-responders/decontamination/ecbc_guide_masscasualtydecontam_0813.pdf?la=en. 2013. Accessed June 19, 2019.

18. Leary AD, Schwartz MD, Kirk MA, Ignacio JS, Wencil EB, Cibulsky SM. Evidence-based patient decontamination: an integral component of mass exposure chemical incident planning and response. Disaster Med Public Health Prep. 2014; 8(3): 260-266. doi: 10.1017/dmp.2014.41

19. Pinette J, Pearce, L., Larcombe B, Vaughan A. CBRNE and hazardous materials self-care decontamination workshop report. Web site. https://simtec.jibc.ca/sites/default/files/HazardsWorkshopReportFinalMarch27.pdf. 2014.

20. Chilcott RP, Larner J, Matar H.The United Kingdom's initial operational response and specialist operational response to CBRN and HazMat incidents: A primer on decontamination protocols for healthcare professionals. Emerg Med J. 2019; 36: 117-123. doi: 10.1136/emermed-2018-207562

21. Macintyre AG, Christopher GW, Eitzen E, Gum R; Weir S, DeAtley C, Tonat K, Barbera JA. Weapons of mass destruction events with contaminated casualties: Effective planning for health care facilities. JAMA. 2000; 283(2): 242-249. doi: 10.1001/ jama.283.2.242 
22. Georgopoulos PG, Fedele P, Shade P, et al. Hospital response to chemical terrorism: Personal protective equipment, training, and operations planning. Am J Ind Med. 2004; 46: 432-445. doi: 10.1002/ajim.20075

23. Harvard school of public health. Emergency preparedness and response exercise program. strategies for first receiver decontamination: A collection of tactics to assist hospitals address common challenges associated with all-hazards decontamination of patients. Web site. https://cdn1.sph.harvard.edu/wp-content/uploads/ sites /1435/2013/01/Strategies-for-First-Receiver-Decontamination-2013.pdf. 2013. Accessed June 19, 2019.

24. Robert PC. Managing mass casualties and decontamination. Environ Int. 2014; 72: 37-45. doi: 10.1016/j.envint.2014.02.006 